\title{
Reliability of chromogenic in situ hybridization for detecting HER-2 gene status in breast cancer: comparison with fluorescence in situ hybridization and assessment of interobserver reproducibility
}

\author{
Yun Gong, Michael Gilcrease and Nour Sneige \\ Department of Pathology, The University of Texas MD Anderson Cancer Center, Houston, TX, USA
}

\begin{abstract}
Accurate determination of HER-2 status is important in the management of patients with breast cancer, especially in determining their eligibility for trastuzumab therapy. Fluorescence in situ hybridization (FISH) has been regarded as the gold standard method for detecting HER-2 gene amplification. Recently, chromogenic in situ hybridization (CISH), in which HER-2 is detected by a peroxidase reaction and the gene copies are determined by regular bright-field microscopy, has emerged as a potential alternative to FISH. However, this method requires validation before it can be adopted into clinical practice. In this study, we evaluated 80 cases of invasive breast carcinoma by CISH, compared the results with those obtained by FISH, and assessed interobserver reproducibility among three observers. We found that agreement among the three pathologists on the CISH-determined HER-2 status was achieved in 73 cases (91\%), all of which had results matching the corresponding FISH results: 54 nonamplified and 19 amplified. Of the 19 amplified cases, 13 were scored unanimously as high-level amplification; six had a minor scoring discrepancy (ie, low-level vs high-level amplification). A major scoring discrepancy (ie, nonamplification vs amplification) was found in the remaining seven cases, three of which were amplified and four of which were nonamplified by FISH. Two of the latter cases had a polysomy of chromosome 17 . The cases that caused scoring difficulty were those with an equivocal or borderline signal number against a high background. Overall, there was nearly perfect agreement between the $\mathrm{CISH}$ and corresponding FISH results, and interpretation of CISH results were highly reproducible among the three pathologists. We conclude that, in general, HER-2 status can be reliably assessed by CISH. Confirmatory FISH is recommended in cases with equivocal or borderline CISH copy numbers.
\end{abstract}

Modern Pathology (2005) 18, 1015-1021. doi:10.1038/modpathol.3800432; published online 29 April 2005

Keywords: HER-2; breast carcinoma; chromogenic in situ hybridization; fluorescence in situ hybridization; interobserver; reproducibility

The HER-2 oncogene, located on chromosome 17q21, encodes a transmembrane tyrosine kinase receptor with extensive homology to the epidermal growth factor receptor. ${ }^{1}$ Amplification of the HER-2 gene or overexpression of the HER-2 protein has

Correspondence: Dr N Sneige, MD, Department of Pathology, Unit 53, The University of Texas MD Anderson Cancer Center, 1515 Holcombe Blvd., Houston, TX 77030, USA.

E-mail: nsneige@mdanderson.org

This study was presented in part at the 93rd Annual Meeting of the United States and Canadian Academy of Pathology, Vancouver, BC, Canada, 2004.

Financial disclosure: The authors have no connection to any companies or products mentioned in this article.

Received 6 February 2005; revised 15 March 2005; accepted 17 March 2005; published online 29 April 2005 been identified in 10-30\% of breast cancers. HER-2 is currently considered not only as a marker of poor prognosis in node-positive patients, but also as a useful determinant of susceptibility to chemotherapy. ${ }^{2-7}$ With the availability of trastuzumab (Herceptin), a humanized monoclonal antibody against the extracellular portion of the HER-2 receptor protein, there has been a growing clinical demand for accurate determination of HER-2 status, as the presence of gene amplification or protein overexpression is by far the major criterion for trastuzumab eligibility.

Various methods are available to determine the HER-2 status in patients with breast cancer, including assays to evaluate gene amplification, mRNA level and protein expression. ${ }^{8,9}$ Fluorescence in situ 
hybridization (FISH) and immunohistochemical study are the two assays currently used to determine the HER-2 status in routine practice with FISH to be more specific and reproducible than immunohistochemical analysis. Clinicopathologic correlations have shown a strong association between the HER2 gene amplification detected by FISH and the clinical response to trastuzumab therapy. ${ }^{8,10}$ However, the main obstacle to the popularization of FISH is the need of additional equipment for analysis such as dark-field fluorescence microscopy and multiband fluorescence filters, which are not widely available in pathology laboratories.

Recently, chromogenic in situ hybridization (CISH) has emerged as a potential alternative to FISH. In CISH, the HER-2 gene is detected by a peroxidase reaction that allows concomitant histopathological evaluation of tissues with a standard bright-field microscope. So far, a number of studies have shown generally good concordance between CISH and FISH results, ${ }^{11-18}$ but studies on interobserver reproducibility in interpreting the results of CISH are lacking. Whether CISH is equally reliable as FISH in HER-2 assessment is still a matter of debate, given certain limitations of CISH. $^{8}$

To prove the reliability of this new method, we evaluated the accuracy of CISH in detecting HER-2 gene amplification by comparing CISH results with corresponding FISH results in 80 cases of invasive breast carcinoma and by assessing interobserver reproducibility.

\section{Materials and methods}

\section{Patients and Specimen Collection}

A total of 80 consecutive cases of invasive breast carcinoma with a known HER-2 gene status according to a previous FISH, performed as part of the prognostic/predictive panel for breast carcinoma, were retrieved from the surgical pathology archives at The University of Texas MD Anderson Cancer Center. The age of the patients at diagnosis ranged from 24 to 80 years (median, 52 years). There were 75 invasive ductal carcinomas, two invasive lobular carcinomas and three mixed ductal and lobular carcinomas. The nuclear grade of tumors, according to the modified Black's nuclear grading system, was 1 (low) in three cases, 2 (intermediate) in 41 cases and 3 (high) in 36 cases. CISH was performed on tissue sections using the same formalin-fixed, paraffin-embedded blocks that had been used for FISH.

\section{FISH}

Routinely processed paraffin-embedded tissue sections $(4-\mu \mathrm{m})$ had been analyzed using FISH protocol (Vysis, Downers Grove, IL, USA). Tissue sections were deparaffinized in three changes of fresh xylene for $3 \mathrm{~min}$ each, dehydrated in two changes of $100 \%$ ethanol for $3 \mathrm{~min}$ each and then allowed to air dry. Slides were then placed in a preheated $80^{\circ} \mathrm{C}$ pretreatment reagent (1 $\mathrm{M}$ sodium isothiocyanate; Vysis) for $13 \mathrm{~min}$, rinsed in distilled water for $3 \mathrm{~min}$ and allowed to air dry. Protease digestion was accomplished by placing the slides in a prewarmed $37^{\circ} \mathrm{C}$ protease solution (Vysis) for $13 \mathrm{~min}$. Samples were then rinsed in distilled water for $3 \mathrm{~min}$ and air dried.

The HER-2 DNA probe kit (PathVysion; Vysis) we used contains two DNA probes directly labeled with different fluorescent dyes: the SpectrumOrange fluorophore-labeled HER-2 $(190 \mathrm{~kb})$ specific for the HER-2 gene locus on chromosome 17q11.2-q12, and the SpectrumGreen fluorophore-labeled chromosome enumerator probe $(5.4 \mathrm{~kb})$ targeted to the alpha satellite DNA sequence located at the centromeric region of chromosome 17 (CEP17; 17p11.1-q11.1). All slides were hybridized under identical conditions and with appropriate control tissue.

An aliquot of prediluted LSI HER-2 SpectrumOrange/CEP17 SpectrumGreen (Vysis) was applied to the region of interest on the slide. A coverslip was placed and sealed at the periphery with rubber cement. The slides were placed on a preprogrammed, humidified slide warmer (Hybrite; Vysis) with the following settings: denaturation at $73^{\circ} \mathrm{C}$ for $5 \mathrm{~min}$, and hybridization at $37^{\circ} \mathrm{C}$ for $16 \mathrm{~h}$. After hybridization, the rubber cement was removed, and the coverslip was floated off by soaking the slides in $2 \times$ standard saline citrate buffer with $0.3 \%$ Nonidet P-40 ( $2 \times$ SSC/0.3\% NP-40) at ambient temperature. The slides were incubated in prewarmed $2 \times$ SSC/ $0.3 \% \mathrm{NP}-40$ at $73^{\circ} \mathrm{C}$ for $2 \mathrm{~min}$, immersed in $2 \times \mathrm{SSC} /$ $0.3 \% \mathrm{NP}-40$ at ambient temperature for $1 \mathrm{~min}$, air dried in the dark, counterstained with $7 \mu \mathrm{l}$ of 4,6diamidino-2-phenylindole dihydrochloride (DAPI; Vysis) and coverslipped.

The signals were enumerated using an epifluorescence microscope (Olympus AX70; Melville, NY, USA) fitted with a SpectrumOrange, SpectrumGreen, and DAPI triple-filter set. At least 60 cells were scored in each preparation, and the copy numbers of HER-2 and CEP17 for each cell were recorded. HER-2 was quantified using the ratio of HER-2 to CEP17 signal counts. HER-2 gene amplification was defined as an HER-2 to CEP17 signal ratio $\geq 2.0$. Polysomy of chromosome 17 was defined as the presence of three or more CEP17 signals in $>6 \%$ of the tumor cells evaluated.

\section{CISH}

Unstained 4- $\mu$ m-thick, archival, formalin-fixed and paraffin-embedded tissue sections were deparaffinized in three changes of fresh xylene for $4 \mathrm{~min}$ each, dehydrated in two changes of $100 \%$ ethanol for $4 \mathrm{~min}$ each and then allowed to air dry. The 
slides were then incubated in pretreatment buffer (1 M sodium isothiocyanate; Vysis) in a water bath at $80^{\circ} \mathrm{C}$ for $13 \mathrm{~min}$, washed with distilled water and digested with protease (Vysis). The slides were then washed with distilled water and dehydrated with a graded series of ethanol. An aliquot of the ready-touse, digoxigenin-labeled HER-2 probe (Zymed, South San Francisco, CA, USA) was applied onto the sections; the sections were then coverslipped and sealed with rubber cement. The sample and probe were codenatured in a Hybrite (Vysis) at $74^{\circ} \mathrm{C}$ for $5 \mathrm{~min}$. After overnight hybridization at $37^{\circ} \mathrm{C}$, the rubber cement and the coverslip were removed, and the slides were washed in $2 \times \mathrm{SSC} / 0.3 \% \mathrm{NP}-40$ (Vysis) at $73^{\circ} \mathrm{C}$ for $2 \mathrm{~min}$, followed by three washes in distilled water; the slides were then immersed in absolute methanol with $3 \% \mathrm{H}_{2} \mathrm{O}_{2}$ and incubated with a blocking reagent for $10 \mathrm{~min}$ at an ambient temperature. The hybridization signals were detected after sequential incubations with mouse antidigoxigenin, polymerized horseradish peroxidase anti-mouse antibody and diaminobenzidine, according to the manufacturer's instructions (Zymed). Slides were counterstained with hematoxylin.

Controls were included in each staining run. Positive controls were tissue samples of breast carcinoma shown by FISH to have high levels of HER-2 amplification. Negative controls were tissue samples of breast carcinoma and normal breast tissue shown by FISH to be negative for HER-2 amplification.

CISH results were independently evaluated by the pathologists (YG, MG, and NS) using light microscope under a $40 \times$ dry objective. Based on previous studies, ${ }^{12,17}$ tumors with no HER-2 amplification typically had one to two brown intranuclear spots per nucleus when diploid, or three to five spots when chromosomally polyploid. Thus, nonamplification was defined as one to five brown intranuclear signals per nucleus, low-level amplification as six to 10 signals per nucleus in more than $50 \%$ of the tumor cells and high-level amplification as more than 10 signals or as large signal clusters in more than $50 \%$ of the tumor cells.

\section{Statistical Analysis}

CISH results were compared with FISH results for the same tumors. Interobserver reproducibility of CISH results was evaluated by a pairwise comparison. Concordance and the Cohen kappa coefficient were calculated using statistical software StatXact-4 Version 4.0.1. The relationship between the kappa value and the level of agreement was suggested by Landis and Koch:19 a kappa value of $0.00-0.20$ indicates slight agreement; 0.21-0.40, fair agreement; 0.41-0.60, moderate agreement; 0.61-0.80, substantial agreement; and 0.81-1.00, almost perfect agreement.

\section{Results}

Of the 80 cases of invasive breast carcinoma, FISH detected HER-2 amplification in 22 cases and nonamplification in 58 cases. Agreement among the three pathologists on the CISH-determined HER2 status was achieved in 73 cases (91\%), all of which had HER-2 status matching with the corresponding FISH results: 19 amplified and 54 nonamplified (Figure 1a and b). Among the 19 amplified cases, 13 were scored unanimously as high-level amplification; while, six cases had minor interobserver disagreement. The differences in these six cases were between low-level and high-level amplification; therefore overall HER-2 status was not changed (Table 1). A major interobserver scoring discrepancy (ie, between nonamplification and amplification) was found in the remaining seven cases (9\%), three shown by FISH to be amplified and four shown to be nonamplified. Two of the nonamplified cases displayed polysomy of chromosome 17, with average CEP17 copy numbers of 4.08 and 3.23 , respectively (Table 1).

When the interpretation of CISH results made by each pathologist was compared with the corresponding FISH results, a major discrepancy in HER-2 status was found in two $(2.5 \%)$, three $(3.8 \%)$ and four $(5.0 \%)$ cases, respectively. Nonetheless, statistical analysis showed that the CISH results as interpreted by each pathologist agreed near perfectly with the corresponding FISH results and that CISH scoring was highly reproducible among the three pathologists (Tables 2 and 3).

Enumeration of the CISH signal was usually straightforward when the signal intensity was high with a low background or in cases with high-level amplification with clusters of multiple gene copies. The difficult cases were those with a signal number that was borderline between nonamplification and low-level amplification (mostly 4-8 signals per nucleus), and cases with equivocal signals of low contrast against a high background. These difficulties were encountered in six of the seven cases $(86 \%)$ with a major scoring discrepancy (Table 1 and Figure 1c and d). Intratumoral variation in the CISH signal distribution was found in two cases in which tumor cells without HER-2 amplification focally abutted tumor cells with amplification (low-level and/or high-level) (Table 1 and Figure 2a-c). The corresponding FISH in both cases showed amplification, but without detecting significant heterogeneity.

\section{Discussion}

An unequivocal determination of HER-2 status is crucial for appropriate management of patients with breast cancer. Although FISH is currently regarded as the gold standard method for detecting HER-2 amplification, CISH has recently been gaining in popularity. Proponents of the CISH technique 
believe that CISH is technically simpler and more straightforward, while it still adheres to the main principles of FISH; CISH allows detection of HER-2
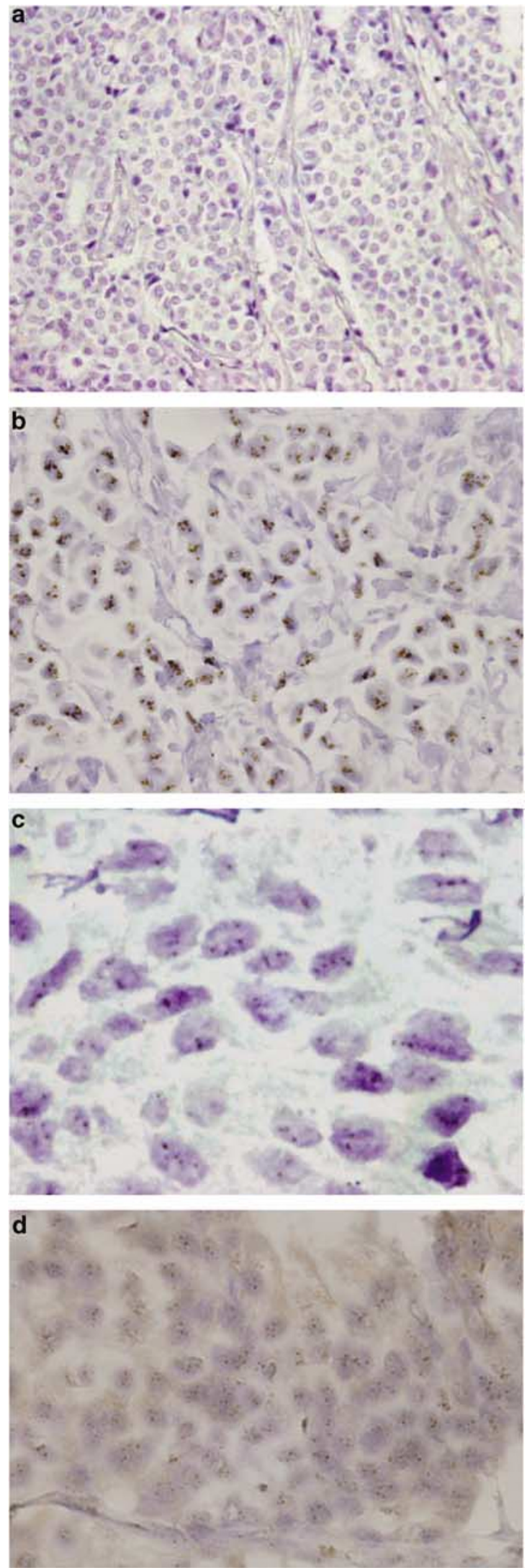

amplification with a conventional peroxidase reaction, enumeration of gene copy number with simultaneous histologic examination by regular bright-field microscopy, and permanent storage of slides. ${ }^{12}$ Previous studies have shown an overall good agreement between CISH and FISH results

Table 1 Chromogenic in situ hybridization (CISH) scoring discrepancies among the three observers and between CISH and corresponding fluorescence in situ hybridization (FISH) results

\begin{tabular}{|c|c|c|c|c|c|}
\hline \multirow[t]{2}{*}{ Discrepancy } & \multirow[t]{2}{*}{ Case } & \multicolumn{3}{|c|}{ CISH scores of the three observers } & \multirow[t]{2}{*}{ Fish } \\
\hline & & $A$ & $B$ & $C$ & \\
\hline \multirow[t]{6}{*}{ Minor $^{\mathrm{a}}$} & 1 & $6-10$ & $6-10$ & $>10$ & 7.08 \\
\hline & 2 & $>10$ & $6-10$ & $6-10$ & 5.63 \\
\hline & 3 & $>10$ & $6-10$ & $>10$ & 9.17 \\
\hline & 4 & $>10$ & $6-10$ & $6-10$ & 3.31 \\
\hline & 5 & $6-10$ & $>10$ & $>10$ & 11.76 \\
\hline & 6 & $6-10$ & $6-10$ & $>10$ & 3.08 \\
\hline \multirow[t]{8}{*}{ Major $^{b}$} & 7 & $\leq 5$ & $6-10$ & $\leq 5$ & 0.70 \\
\hline & 8 & $\leq 5$ & $\leq 5$ & $6-10$ & 1.26 \\
\hline & 9 & $6-10$ & $\leq 5$ & $6-10$ & $1.81^{\mathrm{C}}$ \\
\hline & 10 & $6-10$ & $\leq 5$ & $6-10$ & $1.34^{\mathrm{d}}$ \\
\hline & 11 & $>10$ & $\leq 5$ & $>10$ & 2.95 \\
\hline & 12 & $\mathrm{~V}$ & $\mathrm{~V}$ & $\mathrm{~V}$ & 4.66 \\
\hline & & (favor 6-10) & $($ favor $\leq 5)$ & (favor 6-10) & \\
\hline & 13 & $6-10$ & $\begin{array}{c}\mathrm{V} \\
(\text { favor } \leq 5)\end{array}$ & $\begin{array}{c}\mathrm{V} \\
\text { (favor } \leq 5 \text { ) }\end{array}$ & 6.00 \\
\hline
\end{tabular}

${ }^{\mathrm{a}} \mathrm{A}$ minor discrepancy was defined as an interobserver discrepancy in CISH scoring between low-level and high-level amplification of the HER-2 gene without changing the overall amplification status.

${ }^{\mathrm{b}} \mathrm{A}$ major discrepancy was defined as a CISH scoring discrepancy between nonamplification and amplification.

${ }^{\mathrm{C}}$ Polysomy of chromosome 17 with a CEP17 copy number of 4.08 .

${ }^{\mathrm{d}}$ Polysomy of chromosome 17 with a CEP17 copy number of 3.23 .

$\mathrm{V}$, intratumoral variation in scoring with nonamplified regions abutting focal amplified areas.

Table 2 Agreement between chromogenic in situ hybridization (CISH) results as interpreted by each observer and corresponding fluorescence in situ hybridization (FISH) results

\begin{tabular}{lll}
\hline \multirow{2}{*}{ Observer } & \multicolumn{2}{c}{ Agreement between CISH and FISH } \\
\cline { 2 - 3 } & Concordance $\left(C I^{\mathrm{a}}\right)$ & Kappa value $\left(C I^{\mathrm{a}}\right)$ \\
\hline $\mathrm{A}$ & $0.96(0.90-0.99)$ & $0.91(0.80-1)$ \\
$\mathrm{B}$ & $0.95(0.85-0.98)$ & $0.87(0.75-0.99)$ \\
$\mathrm{C}$ & $0.94(0.86-0.97)$ & $0.85(0.71-0.98)$
\end{tabular}

${ }^{\mathrm{a}} 95 \%$ confidence interval.

Figure 1 CISH detection of the HER-2 oncogene in invasive breast carcinomas. (a) carcinoma with no amplification, (b) carcinoma with high-level amplification showing clusters of multiple gene copies. (c), carcinoma with borderline signal numbers (4-8 signals per nucleus). The corresponding FISH revealed no HER-2 amplification, but with polysomy of chromosome 17. (d) Carcinoma with equivocal signals of low contrast against a high background. The corresponding FISH revealed no HER-2 amplification. 
Table 3 Pairwise comparison between observers' interpretation of CISH results

\begin{tabular}{lll}
\hline \multirow{2}{*}{ Observer } & \multicolumn{2}{c}{ Agreement between two observers } \\
\cline { 2 - 3 } & Concordance $\left(C I^{\mathrm{a}}\right)$ & Kappa value $\left(C I^{\mathrm{a}}\right)$ \\
\hline $\mathrm{A}-\mathrm{B}$ & $0.94(0.86-0.97)$ & $0.84(0.71-0.98)$ \\
A-C & $0.98(0.91-0.99)$ & $0.94(0.86-1)$ \\
B-C & $0.94(0.86-0.97)$ & $0.85(0.71-0.98)$
\end{tabular}

${ }^{\mathrm{a}} 95 \%$ confidence interval.

$(84-100 \%) .{ }^{11-18}$ In this study, we found that the three pathologists agreed on the CISH scoring in $91 \%$ of cases, and all of these cases showed complete agreement between CISH and FISH results. Furthermore, our data showed near-perfect agreement between the FISH and the CISH results interpreted by each pathologist. The high interobserver reproducibility validated the reliability of this method for assessing HER-2 status. To date, only one study that examined interobserver reproducibility in interpretations of HER-2 status showed a high level of agreement; however, the investigators used bright-field, gold-based autometallographic in situ hybridization, ${ }^{20}$ a method similar but not identical to peroxidase-based CISH.

Discrepancy in results between CISH and FISH were encountered in few of our cases, most probably because of the differences in scoring strategies used for the two methods and the use of CEP17 as an internal control in the FISH method. The cutoff number for determining oncogene amplification by CISH was empirically defined as six copies per cell based on the observation that most cases with polysomy of chromosome 17 fall into three to five signals per nucleus. ${ }^{12,17}$ However, aneusomy of chromosome 17 is common in breast cancer ${ }^{12,18,21-24}$ and polysomy with $>5$ copies of chromosome 17 per nucleus is not rare. ${ }^{17}$ These cases would be scored erroneously as low amplification while there is no actual amplification present. ${ }^{25}$ On the other hand, using this cutoff could ignore the amplification of four to five oncogene copies per cell when monosomy copies of chromosome 17 is present. These problems occur mainly because the current CISH technique is based on single-color detection of one probe without simultaneous centromere counts and is thereby unable to evaluate numerical alterations of chromosome 17. Indeed, studies have shown that the majority of discrepant cases between CISH and FISH results were due to polysomy of chromosome $17 \cdot{ }^{14,17}$ In the present study, we found only two cases with polysomy of chromosome 17 that resulted in CISH scores of low-level amplification, whereas the corresponding FISH results showed no amplification (Table 1). Since polysomy of chromosome 17 contributed to some of the scoring discrepancies, it is important, prior to
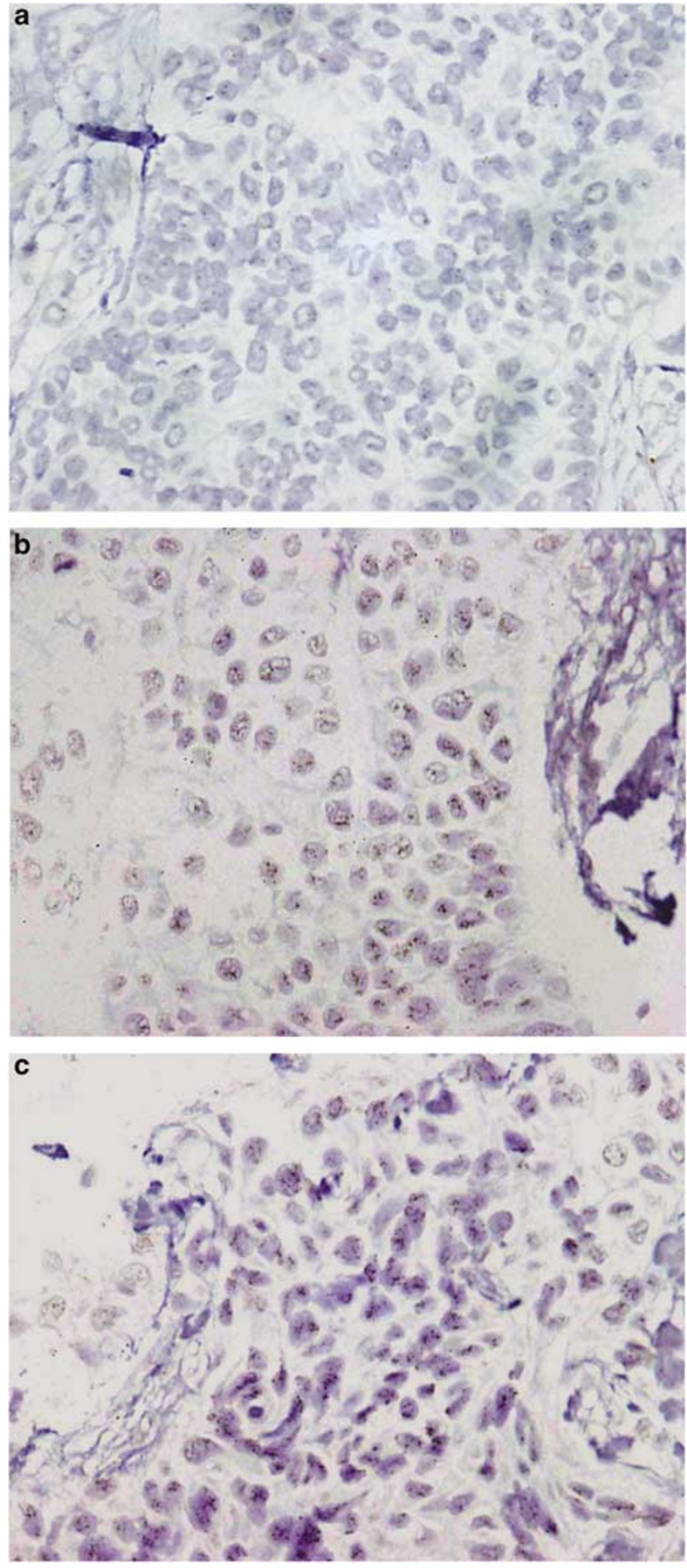

Figure 2 Carcinoma with intratumoral variation in CISH signal distribution with nonamplified regions (a), abutting foci of lowlevel amplified (b), and high-level amplified areas (c).

therapy, to evaluate chromosome 17 copy number in tumors with borderline CISH results. Two-color FISH using probes detecting both HER-2 and CEP17 is ideal for this purpose. Alternatively, the use of a reference probe for chromosome 17 on adjacent 
tissue sections for CISH analysis might achieve similar results, as advocated by some authors. ${ }^{17,18}$

Although enumeration of CISH signals was in most cases straightforward, scoring signals that were borderline between nonamplification and low-level amplification were more challenging. The difficulty can be implicated by a low signal intensity and a high background (Figure 1c and $\mathrm{d}$ ). These technical problems have been previously reported ${ }^{8,12,14,26}$ and can cause misinterpretation or noninterpretable CISH results. ${ }^{17,25,27}$ Preanalytical factors, including variation in fixation or processing conditions, the thickness of the tissue sections, and the level of endogenous peroxidase are additional factors that may affect the quality of CISH technique. ${ }^{13,15,18,27}$ Gold-facilitated autometallographic bright-field in situ hybridization has been reported to enhance signal detection, ${ }^{28}$ but this methodology is complicated and not yet widely available. Heat pretreatment and digestion with pepsin have also been reported to improve signal detection. ${ }^{18,25}$ However, we did not observe much signal enhancement when we used these techniques. In contrast, when using FISH, we found rather bright and distinct signals in these cases.

Interobserver discrepancy in CISH scoring also occurred in cases with intratumoral signal variation. Using immunohistochemical staining, intratumoral heterogeneity of HER-2 expression has been reported in less than $5 \%$ of invasive breast carcinomas. ${ }^{29-32}$ Such heterogeneity is frequently observed in cases with low-level HER-2 overexpression. In the present study, two cases exhibited areas of no HER-2 amplification abutting foci of low-level and/or highlevel amplification (Table1 and Figure 2). Such field variation has been seen in other studies with $\mathrm{CISH} ;{ }^{17,28}$ presumably because larger areas of the tumor may be examined with CISH, this technique is able to detect subclones that might be missed under high-power dark-field microscopy used in FISH..$^{33}$ However, in view of the corresponding FISH results that showed HER-2 amplification but without signal variation in both cases, technical problems that cause false negativity might be another possibility.

In summary, our examination of 80 archival breast cancer specimens confirmed that HER-2 status can be reliably determined by CISH in most cases. Cases with equivocal or borderline HER-2 gene copy numbers may be encountered, although generally they are few in number. Given the importance of an accurate assessment of HER-2 status for selecting therapy, confirmatory FISH using dual probes is recommended in these cases.

\section{Acknowledgements}

We thank Emily Tarco for her skillful technical assistance and Sijin Wen for statistical analysis.

\section{References}

1 Popescu NC, King CR, Kraus MH. Localization of the human erbB-2 gene on normal and rearranged chromosomes 17 to bands q12-21.32. Genomics 1989;4: 362-366.

2 Berns EM, Foekens JA, van Staveren IL, et al. Oncogene amplification and prognosis in breast cancer: relationship with systemic treatment. Gene 1995;159:11-18.

3 Leitzel K, Teramoto Y, Konrad K, et al. Elevated serum c-erbB-2 antigen levels and decreased response to hormone therapy of breast cancer. J Clin Oncol 1995;13:1129-1135.

4 Ross JS, Fletcher JA. The HER-2/neu oncogene in breast cancer: prognostic factor, predictive factor, and target for therapy. Stem Cells 1998;16:413-428.

5 Quenel N, Wafflart J, Bonichon F, et al. The prognostic value of c-erbB2 in primary breast carcinomas: a study on 942 cases. Breast Cancer Res Treat 1995;35: 283-291.

6 Slamon DJ, Clark GM, Wong SG, et al. Human breast cancer: correlation of relapse and survival with amplification of the HER-2/neu oncogene. Science 1987;235:177-182.

7 Slamon DJ, Godolphin W, Jones LA, et al. Studies of the HER-2/neu proto-oncogene in human breast and ovarian cancer. Science 1989;244:707-712.

8 Ross JS, Fletcher JA, Bloom KJ, et al. HER-2/neu testing in breast cancer. Am J Clin Pathol 2003;120(Suppl): S53-S71.

9 van de Vijver M. Emerging technologies for HER2 testing. Oncology 2002;63(Suppl 1):33-38.

10 Vogel CL, Cobleigh MA, Tripathy D, et al. Efficacy and safety of trastuzumab as a single agent in first-line treatment of HER2-overexpressing metastatic breast cancer. J Clin Oncol 2002;20:719-726.

11 Vera-Roman JM, Rubio-Martinez LA. Comparative assays for the HER-2/neu oncogene status in breast cancer. Arch Pathol Lab Med 2004;128:627-633.

12 Tanner M, Gancberg D, Di Leo A, et al. Chromogenic in situ hybridization: a practical alternative for fluorescence in situ hybridization to detect HER-2/neu oncogene amplification in archival breast cancer samples. Am J Pathol 2000;157:1467-1472.

13 Arnould L, Denoux Y, MacGrogan G, et al. Agreement between chromogenic in situ hybridisation (CISH) and FISH in the determination of HER2 status in breast cancer. Br J Cancer 2003;88:1587-1591.

14 Gupta D, Middleton LP, Whitaker MJ, et al. Comparison of fluorescence and chromogenic in situ hybridization for detection of HER-2/neu oncogene in breast cancer. Am J Clin Pathol 2003;119:381-387.

15 Dandachi N, Dietze O, Hauser-Kronberger C. Chromogenic in situ hybridization: a novel approach to a practical and sensitive method for the detection of HER2 oncogene in archival human breast carcinoma. Lab Invest 2002;82:1007-1014.

16 Park K, Kim J, Lim S, et al. Comparing fluorescence in situ hybridization and chromogenic in situ hybridization methods to determine the HER-2/neu status in primary breast carcinoma using tissue microarray. Mod Pathol 2003;16:937-943.

17 Isola J, Tanner M, Forsyth $\mathrm{A}$, et al. Interlaboratory comparison of HER-2 oncogene amplification as detected by chromogenic and fluorescence in situ hybridization. Clin Cancer Res 2004;10:4793-4798. 
18 Zhao J, Wu R, Au A, et al. Determination of HER2 gene amplification by chromogenic in situ hybridization (CISH) in archival breast carcinoma. Mod Pathol 2002;15:657-665.

19 Landis JR, Koch GG. The measurement of observer agreement for categorical data. Biometrics 1977;33: 159-174.

20 Tubbs R, Skacel M, Pettay J, et al. Interobserver interpretative reproducibility of GOLDFISH, a first generation gold-facilitated autometallographic bright field in situ hybridization assay for HER-2/neu amplification in invasive mammary carcinoma. Am J Surg Pathol 2002;26:908-913.

21 Wang S, Hossein Saboorian M, Frenkel EP, et al. Aneusomy 17 in breast cancer: its role in HER-2/neu protein expression and implication for clinical assessment of HER-2/neu status. Mod Pathol 2002;15: 137-145.

22 Pauletti G, Godolphin W, Press MF, et al. Detection and quantitation of HER-2/neu gene amplification in human breast cancer archival material using fluorescence in situ hybridization. Oncogene 1996;13:63-72.

23 Gancberg D, Lespagnard L, Rouas G, et al. Sensitivity of HER-2/neu antibodies in archival tissue samples of invasive breast carcinomas. Correlation with oncogene amplification in 160 cases. Am J Clin Pathol 2000;113: $675-682$.

24 Tirkkonen M, Tanner M, Karhu R, et al. Molecular cytogenetics of primary breast cancer by CGH. Genes Chromosomes Cancer 1998;21:177-184.

25 Isola J, Tanner M. Chromogenic in situ hybridization in tumor pathology. Methods Mol Med 2004;97:133-144.
26 Hopman AH, Claessen S, Speel EJ. Multi-colour brightfield in situ hybridisation on tissue sections. Histochem Cell Biol 1997;108:291-298.

27 Kumamoto H, Sasano H, Taniguchi T, et al. Chromogenic in situ hybridization analysis of HER-2/neu status in breast carcinoma. application in screening of patients for trastuzumab (Herceptin) therapy. Pathol Int 2001;51:579-584.

28 Tubbs R, Pettay J, Skacel M, et al. Gold-facilitated in situ hybridization: a bright-field autometallographic alternative to fluorescence in situ hybridization for detection of Her-2/neu gene amplification. Am J Pathol 2002;160:1589-1595.

29 Masood S, Bui MM. Assessment of Her-2/neu overexpression in primary breast cancers and their metastatic lesions: an immunohistochemical study. Ann Clin Lab Sci 2000;30:259-265.

30 Simon R, Nocito A, Hubscher T, et al. Patterns of Her-2/neu amplification and overexpression in primary and metastatic breast cancer. J Natl Cancer Inst 2001;93:1141-1146.

31 Iglehart JD, Kraus $\mathrm{MH}$, Langton BC, et al. Increased erbB-2 gene copies and expression in multiple stages of breast cancer. Cancer Res 1990;50:6701-6707.

32 Niehans GA, Singleton TP, Dykoski D, et al. Stability of HER-2/neu expression over time and at multiple metastatic sites. J Natl Cancer Inst 1993;85:12301235.

33 Takarabe T, Tsuda H, Okada S, et al. Detection of numerical alterations of chromosome 1 in cytopathological specimens of breast tumors by chromogen in situ hybridization. Pathol Int 2001;51:786-791. 